\title{
53. STELLAR RINGS AND GALACTIC STRUCTURE
}

\author{
J. ISSERSTEDT \\ Astronomisches Institut, Ruhr-Universität, Bochum, Germany
}

\begin{abstract}
Stellar rings are shell type prolate ellipsoidal stellar aggregates. The most important property is the constancy of the minor diameters which allows precise geometric distance determinations up to great distances. New photoelectric and photographic UBV-observations of two stellar rings confirm the reality and the diameter constancy of these objects. All A- and F-stars of a nearby ring in Aquila exhibit an UV-excess, similar to that observed in the very young open cluster NGC 2264.
\end{abstract}

Star formation seems to go on in certain gaseous nebulae (Schmidt-Kaler, 1970). In NGC 6888 we are apparently observing the evolution from a gaseous shell to a stellar ring. A few years ago such stellar rings were discovered in a later stage of their evolution without correlated H II regions (Isserstedt, 1968a).

Most important criteria for the definition of the stellar rings are the symmetry to the major diameter, the small thickness of the ring, which is between $\frac{1}{40}$ of the minor diameter, and $\frac{1}{10}$ of this diameter in the worst cases, and a sharp outer boundary. The ratio of the axes is always smaller than 2.0. The rings contain between 25 and 200 stars; an average value is 70 .

In a systematic search for stellar rings over most of the sky with the Lick Observatory Sky Atlas, and near the galactic plane with the red prints of the Palomar Observatory Sky Survey (incl. the Whiteoak Extension) a total of 1070 rings have been found. The minor diameters are between 0.7 and nearly $4{ }^{\circ}$. The rings have been catalogued with their coordinates, diameters and the position angles of the major axes.

Some characteristics of the stellar rings make it most unlikely that they should be nothing but accidental configurations of stars. There is, for example, a great difference between the distributions of stellar rings and normal field stars up to $21^{\mathrm{m}}$. The rings are much more concentrated to the plane, and the absorption layer, recognizable in the distribution of the rings, indicates that the average distance of the rings is greater than the average distance of the field stars.

Another conspicuous fact is the observation that in the greatest rings which were found in the Palomar Atlas, the density of the luminous stars of the HamburgCleveland Spectral Survey is a dozen times the average density in the field near the galactic plane.

A few stars with known MK-classification or with spectra from the Henry Draper Catalogue were found in stellar rings. With these data it became possible to determine a few distances of stars in rings and even three Hertzsprung-Russell diagrams for the nearest objects. All these observations seemed to confirm the reality of the rings and indicated that the minor diameters of the rings are scattering only a little around a mean value of $7.1 \mathrm{pc}$.

In analogy to the shell type gaseous nebulae the stellar rings are shell type ellipsoidal 
stellar associations whose symmetry axis is the major diameter. The minor diameter of these prolate ellipsoidal shells therefore appears always without perspective shortening in projection to the sphere.

To test whether the rings really have always the same minor diameter, the distance of the rings was determined only from the apparent diameters with the assumption that the true minor diameters are always $7.1 \mathrm{pc}$. This hypothesis led to a picture of the galactic structure up to a distance of $15 \mathrm{kpc}$ (Isserstedt, 1968a, 1969b). This structure, almost free of heliocentric effects, proves the reality of the rings and the constancy of the minor diameters. The scatter of the diameters is not larger than $5 \%$; otherwise the far distant details in this picture would be smeared out. It should be stressed that we do not see any possibility to explain this observational result in another way. The Galaxy is of type $\mathrm{Sb}$, perhaps $\mathrm{Sb}-\mathrm{Sc}$. The scale in the picture of the galactic structure is still rather uncertain and depends on the value for the minor ring diameter. With a minor diameter of $7.1 \mathrm{pc}$ the distance to the galactic centre is $10.8 \mathrm{kpc}$.

At first glance the distance range seems surprisingly large. This is possible, because most rings at great distances from the sun are far distant from the galactic plane. This means, that the light has to pass the thin absorption layer only for 2 or $3 \mathrm{kpc}$. The problem is, therefore, not the visibility of these rings but the fact, that there are rings so far from the galactic plane and that these rings conform to the structure in the plane. Most rings which define the structure are less than $1000 \mathrm{pc}$ distant from the plane, but the rings between $500 \mathrm{pc}$ and $1000 \mathrm{pc}$ contribute an important part of the picture, and there are moreover many rings above $1000 \mathrm{pc}$, which are at least partly real objects and not accidental configurations. In spite of the great scatter around the galactic plane the rings define a flat plane in good agreement with the results from $21 \mathrm{~cm}$-line observations (Isserstedt, 1968b).

The galactic structure in the solar neighbourhood, as shown by the distribution of $\mathrm{H}$ II regions and young open clusters (Becker and Fenkart, 1970) is very similar to the local distribution of stellar rings (Isserstedt, 1969b).

To investigate the reality of individual stellar rings and to determine the mean value of the minor diameter more accurately, we began a program of photoelectric and photographic UBV-photometry for several objects. The first results for ring No. 373 show that it is real and that the minor diameter is within the errors of the method identical with the mean value of $7.1 \mathrm{pc}$. The distance of this ring is nearly $3 \mathrm{kpc}$. A detailed analysis is difficult because the absorption is strong and not homogeneous in the field (Isserstedt and Schmidt-Kaler, 1969).

To determine properties of stellar rings, it is necessary to observe the nearest objects with small reddenings and bright stars. Therefore, the Aquila ring, one of the nearest objects, was observed photoelectrically in UBV. The reddening is 0.08 only and homogeneous all over the ring. The distance is $240 \mathrm{pc}$, the minor diameter $7.4 \mathrm{pc}$. There is a clear UV-excess for all A- and F-stars, indicating that the stars are possibly still contracting to the main sequence. The same effect can be seen in the two colour diagrams of the central region of the very young open cluster NGC 2264 (Walker, 1956) and of the nearby stellar ring in Orion (Isserstedt, 1969a). 
We think that these UV-excesses are further evidence for the reality of the stellar rings because such systematic effects are not expected in accidental stellar configurations.

\section{References}

Becker, W. and Fenkart, R. P.: 1970, IAU Symposium No. 38, p. 205.

Isserstedt, J.: 1968a, Veröff. Bochum, No. 1, 1.

Isserstedt, J.: 1968b, Veröff. Bochum, No. 1, 121.

Isserstedt, J.: 1969a, Astron. Astrophys. 3, 210.

Isserstedt, J.: 1969b, in preparation.

Isserstedt, J. and Schmidt-Kaler, T.: 1969, in preparation.

Schmidt-Kaler, T.: 1970, IAU Symposium No. 38, p. 284.

Walker, M. F.: 1956, Astrophys. J. Suppl. Ser. 2, 365.

\section{Discussion}

B. J. Bok: Why are no globular clusters observed on the other side of the galactic center while the ring-spiral system comes through apparently undisturbed by cosmic dust?

What is your response to the paper by David Crampton, who claims that the dispersions in radial velocity in the Aquila and Orion rings are too great to make them stable?

Isserstedt: (1) Nearly no rings are found behind the galactic centre. From 87 rings with distances $R>R_{0}$ between longitudes $0^{\circ}$ and $10^{\circ}$ only 9 objects are less than $500 \mathrm{pc}$ distant from the plane. No ring at all could be found near the galactic centre up to a distance $R=500 \mathrm{pc}$. The structure of the other side of the galaxy can be observed only because those rings are far distant from the plane.

(2) I cannot discuss Crampton's letter, because no individual data are given. 